\title{
Lightweight Design of Control Arm Combining Load Path Analysis and Biological Characteristics
}

\author{
Fenghe $\mathrm{Wu}^{1}$, Zhaohua Wang ${ }^{2}$, Dezhuang Song ${ }^{3}$, Hui Lian ${ }^{4}$ \\ ${ }^{1}$ School of Mechanical Engineering, Yanshan University, Qinhuangdao, China, e-mail: risingwu@ysu.edu.cn \\ ${ }^{2}$ School of Mechanical Engineering, Taiyuan University of Science and Technology, Taiyuan, China, e-mail: \\ wangzhaohua@tyust.edu.cn \\ ${ }^{3}$ School of Mechanical Engineering, Yanshan University, Qinhuangdao, China, e-mail: 1084671626@qq.com \\ ${ }^{4}$ School of Mechanical Engineering, Yanshan University, Qinhuangdao, China, e-mail: godzzz@ stumail.ysu.edu.cn
}

\begin{tabular}{l}
\hline \hline Article Info \\
\hline Article history: \\
Received December 22, 2021 \\
Revised January 20, 2022 \\
Accepted January 21, 2022 \\
\hline
\end{tabular}

\section{Keywords:}

Control arm,

Bionic design,

Load path analysis,

Lightweight.

\begin{abstract}
The existing bionic design methods are based on the similarity theory, but it is difficult to find a bionic prototype with high similarity for the control arm. In this paper, a design method combining load path analysis and biological characteristics is proposed to realize the lightweight of control arm. The load path is used to visualize the load-transferred law in structure and identify the load-bearing performance. Then, the structural improvement suggestions are given and a new control arm with the coupling load of bending moment and pressure is established. Then, the cross-section of wheat-stalk is selected as the cross-section of control arm from four kinds of plants including Bamboo, Wheat-stalk, Juncus, Brazilian Horsetail because of its good bendingresistance and torsion-resistance. The bionic model is designed and the mechanical performance is analyzed and verified by Finite Element Analysis. The results show that the stiffness and strength of the control arm are improved by $65.6 \%$ and $22.5 \%$ respectively, and its weight is reduced by $32.7 \%$, which show the feasibility and efficiency of the proposed method.
\end{abstract}

Copyright (C) 2022 Regional Association for Security and crisis management and European centre for operational research. All rights reserved.

\section{Corresponding Author:}

Zhaohua Wang,

College of Mechanical Engineering, Taiyuan University of Science and Technology, Taiyuan, China.

Email: wangzhaohua@tyust.edu.cn

\section{Introduction}

The control arm is a component for transferring and guiding loads in the vehicle suspension system. Its performance directly affects the stability and reliability of vehicle. In order to ensure stable driving and sensitive steering, the control arm is required to be small enough in weight on the basis of satisfying its stiffness and strength (Heo et al., 2013). Therefore, it is necessary to develop the control arm with high specific stiffness and high specific strength.

At present, the research about lightweight design of control arm focuses on topology optimization (TO) and parameter optimization. Viqaruddina \& Ramana (2017) used topology optimization to optimize a control arm, and the weight was reduced by $30 \%$. Yoo et al. (2017) combined TO and Mean Field Homogenization (MFH) method to optimize a control arm of electric vehicle. Zhang et al. (2017) established the topology optimization objective function of a control arm based on the compromise programming method, and the weight was reduced by $9.3 \%$ while the structural stiffness, strength, as well as the natural frequencies were improved.

The above methods based on numerical analysis (including topology optimization, shape optimization, size optimization, etc.) can realize the lightweight of the control arm, but a rigorous mathematical model needs to 
be established, and stress constraint optimization as one of the classical problems in TO methods still faces many challenges, such as singularity phenomenon and local optimal solution (Cheng et al., 2019). On the other hand, the TO methods based on the black box model are difficult to give the explicitly mechanical guidance for the structural optimization (Suzuki et al. 2020). In addition, lightweight design of control arm by size optimization is to continuously reduce the section parameters. Under the limit lightweight requirements of the control arm, too small section parameters are easy to cause structural instability and even fatigue. Therefore, it is necessary to explore new lightweight design ideas for the control arm.

Biological structures have excellent mechanical performance after hundreds of millions of years of evolution, which provide a lot of creative ideas for structural engineers to solve the lightweight demands, such as bamboo, honeycomb, waterlily, bone and so on (Zhang et al, 2019; Dong \& Zhu, 2016). If the biological characteristics are transferred to the control arm in the design stage, a lot of creative structural features will be provided for the designers. However, the current achievements (Wang et al., 2018; Zhao et al., 2011; Kakoty \& Hazarika, 2013) are based on the similarity theory, usually including structural similarity, functional similarity and load similarity, which leads to the subjectivity in the selection of bionic prototype. In addition, it is difficult to find a bionic prototype with high similarity for those complex structures, especially the skeleton structures such as automobile frame, control arm, steering knuckle, aircraft landing gear, etc.,. So, the application of bionic design in control arm is restricted.

For the above problem, Wang et al. (2020) propose a novel bionic design method by the bio-inspired idea of "main-branch and sub-branch" for skeleton structures. The main-branch is obtained by the load path analysis and structural sub-branch is occupied by the fish-bone structure. The design result shows that the structural stiffness is increased while the weight is reduced. But the selection criterion of bionic prototype is still established from similarity theory. In this study, a new design method of control arm combining load path and biological characteristics is proposed. The structural improvement suggestion is given by load path analysis and the biological prototypes is selected according to the load-bearing type of control arm. A new bionic model is established and its performance is verified by Finite Element Analysis (FEA).

\section{Control arm and design method}

In this paper, a control arm in an automobile is taken as a case study. The installation position is shown in Figure 1, and the structure are shown in Figure 2. The material is aluminum alloy A356 and material properties are given in Table 1. During the driving process, the control arm are in the extreme working condition with the positive load $3062.36 \mathrm{~N}$ in $x$ direction when the wheels hit the side of road. The load and constraint are shown in Figure 2. Two cylindrical holes (B and C) on the right side are directly connected with the vehicle frame, which can be equivalent to fixed constraints, while the cylindrical hole A on the left side is connected with the steering knuckle, which can be equivalent to pin load.

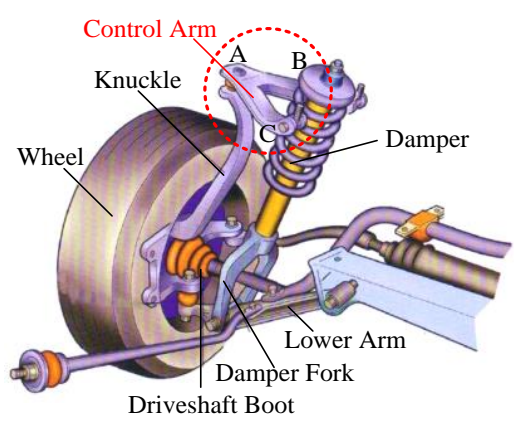

(a)

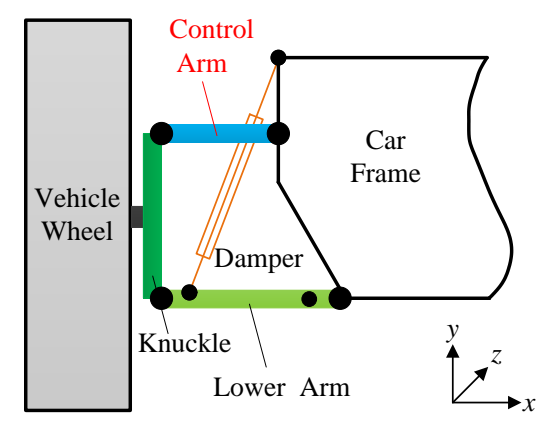

(b)

Figure 1. Suspension system diagram (a) The schematic diagram of suspension system; (b) Brief drawing of suspension system 


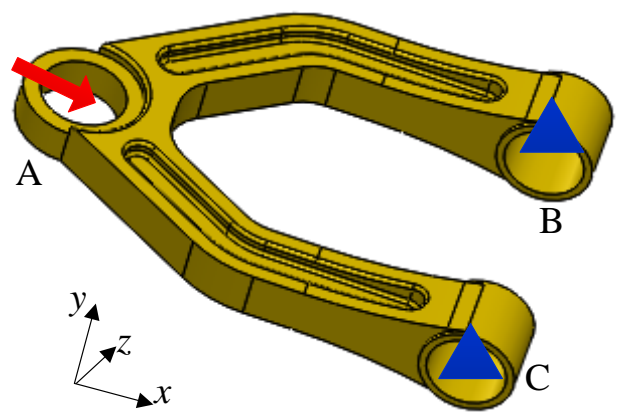

Figure 2. Control arm structure and boundary conditions

Table 1. Material properties of A356

\begin{tabular}{ccccc}
\hline Material & Elastic modulus $(\mathrm{GPa})$ & Poisson's ratio & Density $\left(\mathrm{kg} \cdot \mathrm{m}^{-3}\right)$ & Yield strength $(\mathrm{MPa})$ \\
\hline A356 & 72.4 & 0.33 & 2680 & 220 \\
\hline
\end{tabular}

From Figure 2, the control arm belongs to skeleton structure, and it's difficult to find a biological structure exactly similar to it. So, the traditional bionic method based on the similarity cannot be applied for it. In this study, we developed a practical methodology for control arm to achieve lightweight by combining load path analysis and biological characteristics. The method is divided into the following three steps: (1) The load path of the control arm is visualized and the load-bearing performance is analyzed. Some structural improvement suggestions are given, and the load-bearing type is identified. (2) The appropriate biological prototype is selected as the structural feature of the control arm according to the load-bearing type. (3) Bionic design and performance analysis.

\section{Structural improvement based load path}

\section{1. load path theory}

The load path theory using $U^{*}$ index is based on the concept of relative stiffness proposed by Takahashi (Takahashi et al., 1986). The elastic structure is simplified to the model shown in Figure 3(a), which includes loading point $\mathrm{A}$, supporting point $\mathrm{B}$ and an arbitrary point $\mathrm{C}$. It is assumed that the three points are connected by springs which represent the relative stiffness between any two points of the system (Wang et al., 2018).

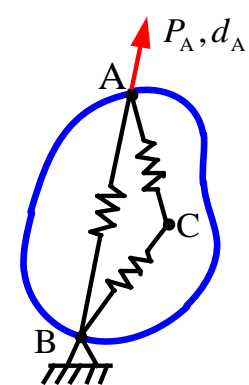

(a)

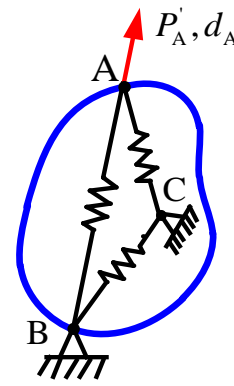

(b)

Figure 3. Simplified model of an elastic structure (a) Original system; (b) Constrained system.

According to the concept of relative stiffness, which is the coupling stiffness between any two points of the structure, the load-displacement relationship among points A, B, and C can be expressed as

$$
\left\{\begin{array}{l}
P_{\mathrm{A}} \\
P_{\mathrm{B}} \\
P_{\mathrm{C}}
\end{array}\right\}=\left[\begin{array}{lll}
K_{\mathrm{AA}} & K_{\mathrm{AB}} & K_{\mathrm{AC}} \\
K_{\mathrm{BA}} & K_{\mathrm{BB}} & K_{\mathrm{BC}} \\
K_{\mathrm{CA}} & K_{\mathrm{CB}} & K_{\mathrm{CC}}
\end{array}\right]\left\{\begin{array}{l}
d_{\mathrm{A}} \\
d_{\mathrm{B}} \\
d_{\mathrm{C}}
\end{array}\right\},
$$

where $K_{i j(i, j=\mathrm{A}, \mathrm{B}, \mathrm{C})}$ is the internal stiffness tensor, $P_{i(i=\mathrm{A}, \mathrm{B}, \mathrm{C})}$ and $d_{i(i=\mathrm{A}, \mathrm{B}, \mathrm{C})}$ represent the load and the displacement vectors, respectively.

Applying load $P_{\mathrm{A}}$ at point A produces displacement $d_{\mathrm{A}}$, the total strain energy $U$ of the system due to the 
external load is calculated according to the strain energy formula:

$U=\frac{1}{2} P_{A} d_{A}$.

Since point B is fixed ( $\left.d_{B}=0\right), P_{A}$ is calculated by the Eq. (1),

$P_{\mathrm{A}}=K_{\mathrm{AA}} d_{\mathrm{A}}+K_{\mathrm{AC}} d_{\mathrm{C}}$.

So, the total strain energy $U$ is:

$U=\frac{1}{2} P_{\mathrm{A}} d_{\mathrm{A}}=\frac{1}{2}\left(K_{\mathrm{AA}} d_{\mathrm{A}}+K_{\mathrm{AC}} d_{\mathrm{C}}\right) d_{\mathrm{A}}$.

In Figure 3(b), any point $\mathrm{C}$ in the structure is fixed and the same displacement $d_{\mathrm{A}}$ is produced by applying load $P_{\mathrm{A}}^{\prime}$ at point A. The total strain energy $U^{\prime}$ is:

$U^{\prime}=\frac{1}{2} P_{\mathrm{A}}^{\prime} d_{\mathrm{A}}=\frac{1}{2}\left(K_{\mathrm{AA}} d_{\mathrm{A}}\right) d_{\mathrm{A}}$.

In order to express the stiffness relationship between an arbitrary point and the loading point in the structure, the dimensionless coefficient $U^{*}$ is defined as:

$U^{*}=\frac{U^{\prime}-U}{U^{\prime}}=\left(1-\frac{2 U}{\left(K_{\mathrm{AC}} d_{\mathrm{C}}\right) \cdot d_{\mathrm{A}}}\right)^{-1}$.

Eq. (6) indicates that the $U^{*}$ only varies with $K_{A C}$ and $U^{*}$ can characterize the internal stiffness between an arbitrary point and loading point of the structure. In an elastic structure, the applied load is mainly transferred through the locations having the strongest coupling stiffness with the loading point. Therefore, connecting nodes with the same stiffness to form a cluster of stiffness contours, the line with the highest $U^{*}$ values which is the ridge line of the $U^{*}$ distribution is the main load path. In a simple case like the structure shown in Figure 4, the main load path is fitted by the $U^{*}$ distribution.

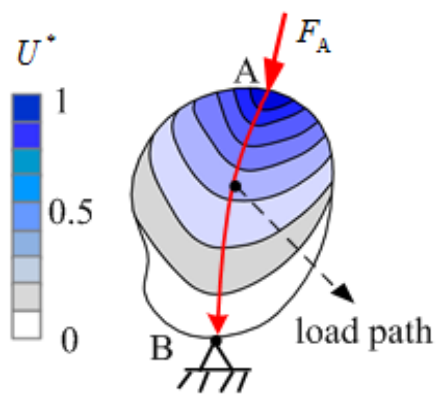

Figure 4. The main load path for a sample structure

The load path method based on $U^{*}$ index has an exceptional advantage for structural analysis (Pejhan et al., 2017; Takahashi et al., 2013) that can give a general awareness regarding the load-bearing performance. Therefore, the load-bearing performance of the structure can be determined by $U^{*}$ along the load path, and the design criteria is defined by authors (Wang et al. 2021). The potential regions need to be optimized and the optimization suggestions can be identified by the criteria: when the $U^{*}$ along the main load path decreases too fast in some regions or when the load path in some regions has a larger curvature, the regions need to be optimized; while the regions with singular $U^{*}$ value, the materials need to be removed or the size needs to be decreased. Taking two flat plates with different shape as an example, the left end of the plate is fixed and a compressive load $F$ is applied to the right end. The $U^{*}$ distribution is calculated and the results are shown in Figure 5, where the black line with arrow represents the main load path. 

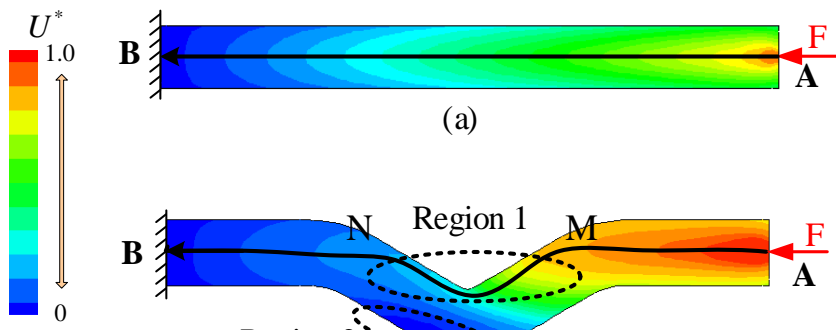

(a)

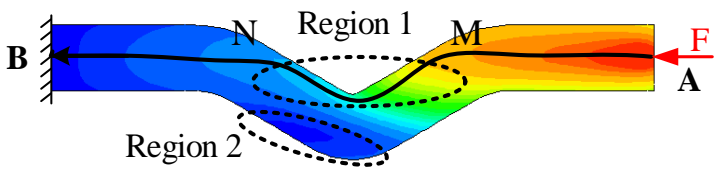

(b)

Figure 5. Load path of flat plate (a) rectangular plate (b) special-shaped plate

The $U^{*}$ along two main load paths is analyzed and the results are shown in Figure 6. Obviously, the load path on the rectangular plate is more uniform and smooth, while the $U^{*}$ value changes abruptly when the load is transferred to the point $\mathrm{M}$ of Region 1 on the special-shaped plate, and then rapidly drops to the point $\mathrm{N}$, then slowly drops to the supporting point. The two points ( $\mathrm{M}$ and $\mathrm{N}$ ) on the load path with abrupt changes of $U^{*}$ value can be reflected by the highest and lowest curvature of $U^{*}$. It is concluded that the $U^{*}$ value decreases too fast in Region 1 (located between $\mathrm{M}$ and $\mathrm{N}$ ) of the special-shaped plate. So, the relative stiffness of Region 1 needs to be improved by increasing the size or materials with lower $U^{*}$ curvatures. Besides, theoretically, the $U^{*}$ decreases continuously along the load path, however, the $U^{*}$ in Region 2 in Figure 5(b) decrease firstly and then increases looking at the locations further away from the loading point. This indicates that the Region 2 with material redundancy has little effect on the structural relative stiffness to loading point A. The smoothness of the load path can be improved by reducing size or materials in Region 2. Moreover, it is the most ideal state to improve the special-shaped structure into a rectangular plate.

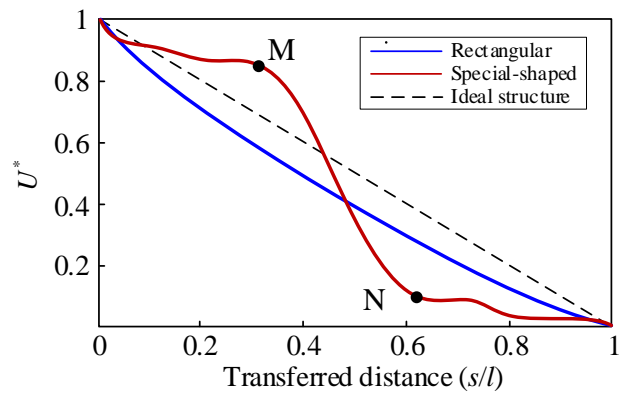

Figure 6. The uniformity of load path

On the one hand, the load path extracted is a concentrated reflection for the load-transferred law in structure, which can indicate the load-bearing types in local region to help to select structural bionic prototype. As shown in Figure 7, the load transfer from load point A to support point B, which passes through arbitrary point $\mathrm{M}, \mathrm{N}$ in turn $(A \rightarrow M \rightarrow N \rightarrow B)$. From point $A$ to $M$, the structure is subjected to the pressure. When the load transfer to the point $\mathrm{M}$, the load path is disturbed by the structural shape and the load-transferred direction rotates anticlockwise, which results in the bending moment from point M to N. Similarly, the load-transferred direction is changed along clockwise at point $\mathrm{N}$, and the load-bearing type becomes coupling load including pressure and bending moment. So, the biological prototype, which is closest to the load-bearing type of the local area of the structure, is selected to carry out the bionic design.

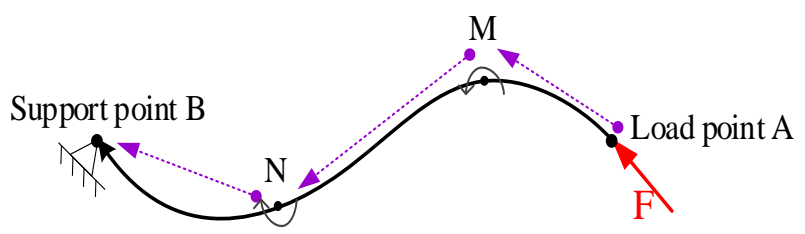

Figure 7. The load type by load path analysis

\subsection{Load path analysis of control arm}

Because the load of control arm is mainly transferred from the loading hole A to the constraint holes B and $\mathrm{C}$, the load path is affected by the shape of the two legs. In order to reduce the calculation time, the model is simplified to a two-dimensional plane and the $U^{*}$ distribution and main load path (red line) is calculated as 
shown in Figure 8. For this kind of skeleton structures, its failure location mainly occurs on the boundary line, so we usually pay more attention to the load-transferred performance of the boundary line. The load-transferred law of the inner side (load path 1 in Figure 8) and the outer side (load path 2 in Figure 8) of the control arm are analyzed respectively. The $U^{*}$ along two load paths are shown in Figure 9.

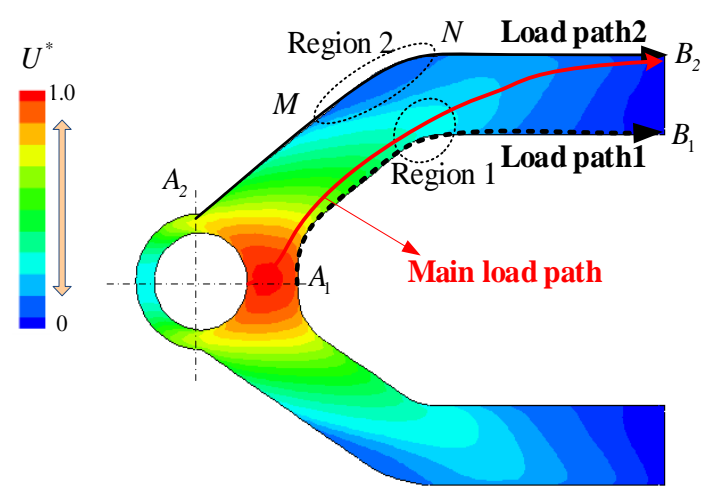

Figure 8. Load path visualization of control arm

The section AM of the control arm mainly bears the coupling load of bending moment and pressure, while the section NB mainly bears the pressure. In theory, we need to select two different biological cross-section characteristics with different load types for bionic design. It can be seen from Figure 9 that in Section AQ of two load paths, the $U^{*}$ of load path 1 is significantly larger than load path 2, which indicates that the stiffness contribution by the inner side of the control arm is greater than that of the outer side before the load is transferred to point Q, and then the stiffness contribution of the outer side is greater from point Q to B. On the load path $1, U^{*}$ in Section AM drops uniformly at a certain rate, while in Section MN (corresponding to Region 1 in Figure 8), the descending rate gradually decreases. This is mainly because the corner of Region 1 hinders the transmission of load, which leads to the load transfer to the outer side of the control arm leg. On the load path 2, the $U^{*}$ firstly decreases from point A to $\mathrm{M}$, then increases from point $\mathrm{M}$ to $\mathrm{N}$, and then decreases to $\mathrm{B}$, which indicate that there is material redundancy and the load transfer can be smoother by reducing the materials in Region 2. Therefore, we can concluded that the unreasonable design of the corner (corresponding to Region 1 and Region 2 in Fig 8) leads to the main load path transfer along the inner side first (Section AM), and then to the outer side (MB). It is necessary to optimize Region 1 and Region 2 to improve the load-transferred performance of the control arm.

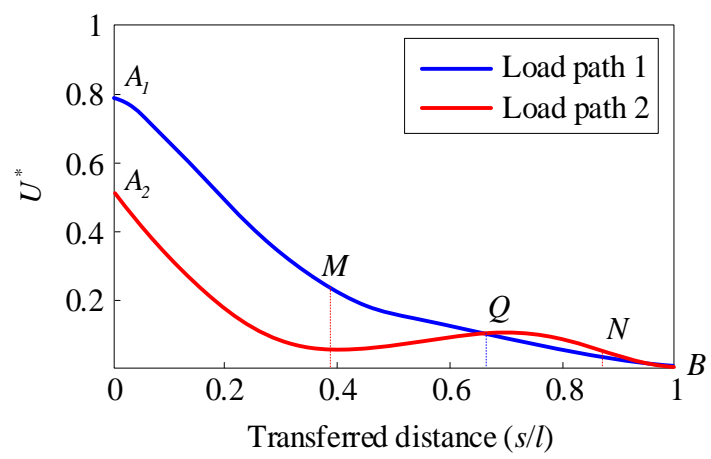

Figure 9. The uniformity of two load paths

\subsection{Load path improvement}

Based on the analysis of Section 3.2, we try to make the load transfer more smoothly. By moving the material in Region 2 to Region 1, the original outline of the control arm is modified to a new outline along the main load path (Figure 8), as shown in Figure 10. The section AB mainly bears the coupling load of bending moment and pressure. 


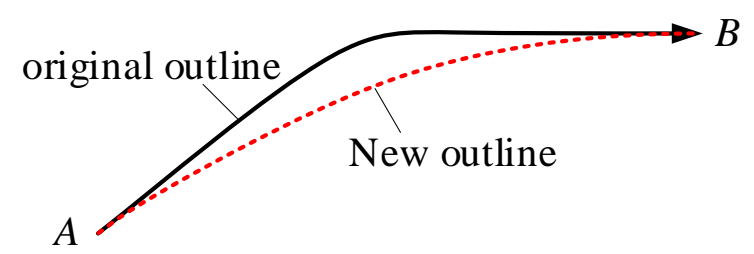

Figure 10. Improved outline based on the load path analysis

According to the new outline, the new model of control arm with same lightweight hole is shown in Figure 11. The weight is reduced from $1.01 \mathrm{~kg}$ to $0.92 \mathrm{~kg}$, accounting for $8.9 \%$. It should be pointed out that the crosssection of the control arm can be designed as a rectangle or a circle, and only the rectangular cross-section is shown here.

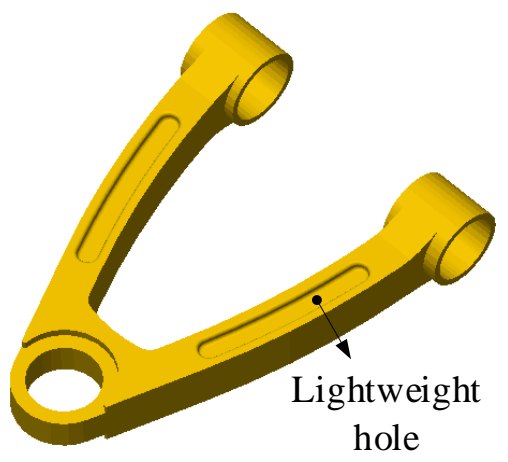

Figure 11. The new model of the control arm

Then, the load path of simplified two-dimensional model is visualized in Figure 12 and the $U^{*}$ along load path is given in Figure 13. It can be seen that in Section AQ of two load paths of new model, the $U^{*}$ of load path 1 is also larger than load path 2, and then the $U^{*}$ value tends to be same from point Q to B. So, the stiffness contribution by the inner side of the control arm is greater than that of the outer side before the load is transferred to point $\mathrm{Q}$, and then the stiffness contribution becomes the same from point $\mathrm{Q}$ to $\mathrm{B}$, which indicate the load-transferred performance becomes more uniform compared with the original model. Compared with the load path 1 of the original model and new model, the change of $U^{*}$ attenuation rate of the new model is more uniform when the load is transferred to $M$, which is mainly because the load transfer in Region 1 is smoother after model modification. Similarity, the comparison of load path 2 show that the $U^{*}$ has always maintained a downward trend for the new model, So, the material redundancy has been eliminated in Region 2 by reducing the materials.

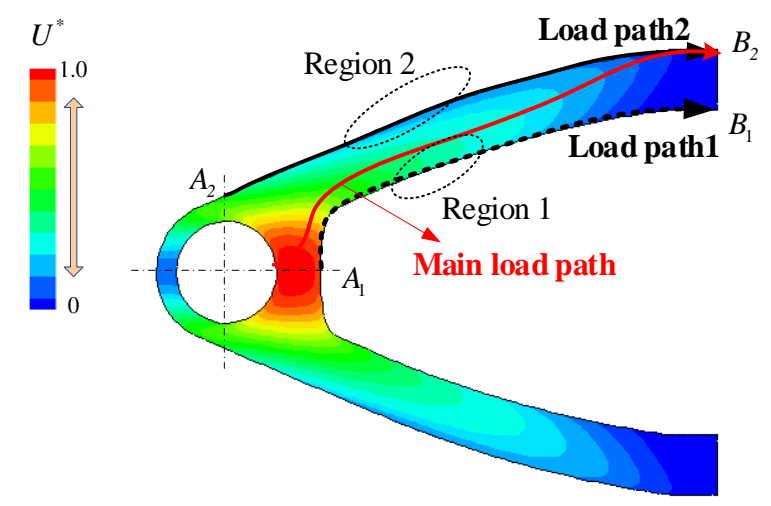

Figure 12. Load path visualization of new control arm 


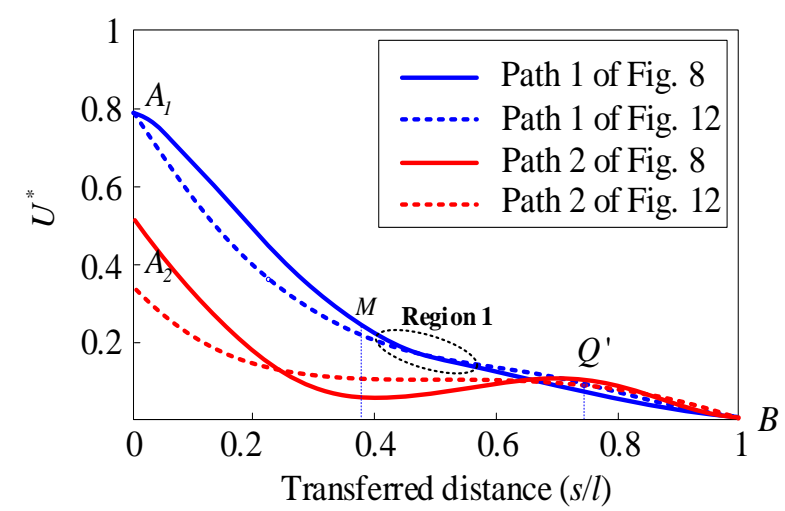

Figure 13. The uniformity comparison of two load paths

To sum up, the load-transferred performance of the new control arm is obviously better than that of the original model, and the load-bearing type is simplified as a coupling load of bending moment and pressure.

\section{Biological cross-section}

Under the influence of heredity and evolution, organisms have gradually formed their own unique cross sections, which provides a great deal of inspiration for the design of control arm. Therefore, the bionic prototype, which is similar to the load-bearing mode, can be selected. For example, the bone mainly bears tension and pressure (Cai \& Shi, 2010), and the plant stem mainly bears bending moment (Waldron \& Harwood, 2010). In this section, the Bamboo, Wheat-stalk, Juncus, Brazilian Horsetail are selected as biological prototypes of control arm. The cross-section features of four kinds of plants are shown in Figure 14.

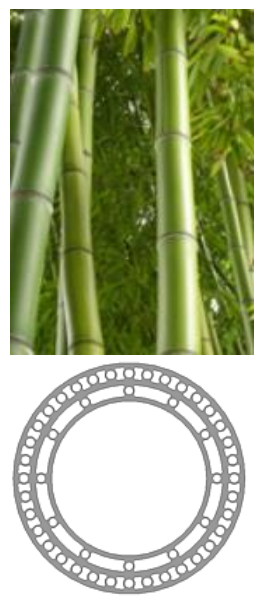

(a)
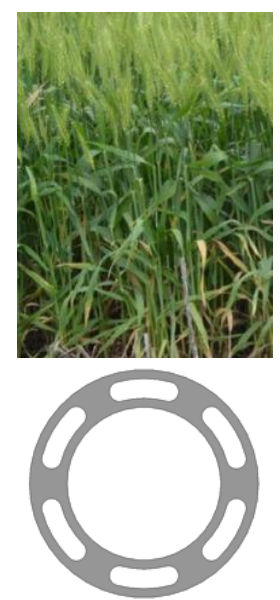

(b)
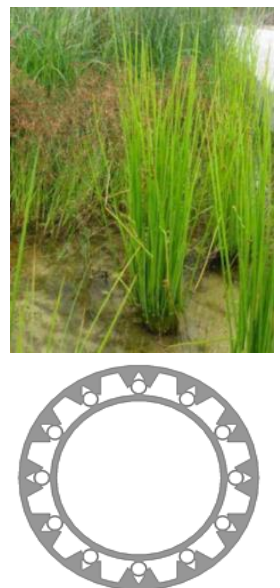

(c)
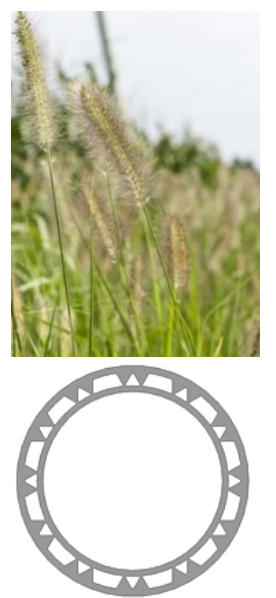

(d)

Figure 14. Four kinds of biological cross-section features (a)Bamboo; (b)wheat-stalk; (c)Juncus; (d)Brazilian Horsetail

In order to determine which load-bearing type is more suitable for the control arm, the simplified CAD models by means of biological cross-section are established respectively in Figure15. The basic dimensions of the cylinder are: the outer diameter is $30 \mathrm{~mm}$, the inner diameter is $10 \mathrm{~mm}$, and the length is $100 \mathrm{~mm}$. The crosssection area of the four kinds of rods should be the same as far as possible to ensure the same weight of the rods.

(a)

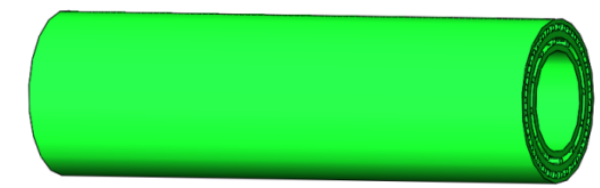

(b)

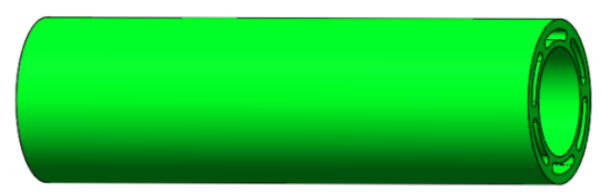


(c)

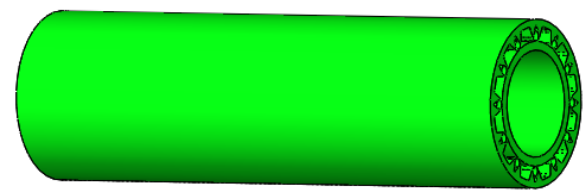

(d)

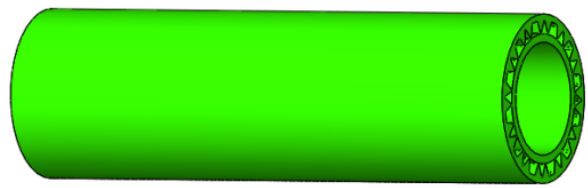

Figure 15. The CAD models of four kinds of bionic model (a)Bamboo; (b)wheat-stalk; (c)Juncus; (d)Brazilian Horsetail

The Finite Element Model is established with same element type and size. And the same load magnitude and direction are taken as boundary condition to verify the pressure-resistance, bending-resistance and torsionresistance characteristics by FEA. The maximum displacement reflecting structural stiffness is calculated as shown in Table 2.

Table 2. The maximum displacement under different load-bearing types (Unit: $\mathrm{mm}$ )

\begin{tabular}{|c|c|c|c|c|c|}
\hline & & Bamboo & wheat-stalk & Juncus & $\begin{array}{l}\text { Brazilian } \\
\text { Horsetail }\end{array}$ \\
\hline $\begin{array}{l}\text { Pressure- } \\
\text { resistance }\end{array}$ & $\mathrm{F}=10000 \mathrm{~N}$ & 0.0183 & 0.0183 & 0.0183 & 0.0179 \\
\hline $\begin{array}{l}\text { Bending- } \\
\text { resistance }\end{array}$ & $\mathrm{F}=10000 \mathrm{~N}$ & 0.8866 & 0.8392 & 0.8795 & 0.8456 \\
\hline $\begin{array}{l}\text { Torsion- } \\
\text { resistance }\end{array}$ & $\mathrm{M}=10000 \mathrm{Nmm}$ & 0.0048 & 0.0045 & 0.0052 & 0.0052 \\
\hline Mass (kg) & N/A & 0.2127 & 0.2132 & 0.2121 & 0.2182 \\
\hline
\end{tabular}

Structural efficiency refers to the structural comprehensive performance per unit weight in the case of meeting load-bearing properties. It is commonly used to evaluate the structural overall performance. In this paper, the specific stiffness efficiency coefficient $\varepsilon_{i}$ is introduced and its calculation formula is shown in Eq. (7). The coefficient represents the structural stiffness of the unit weight. The larger the value, the greater the structural stiffness, which indicates that the material distribution is more reasonable.

$\varepsilon_{i}=\frac{E / d_{i}}{m_{i}} \quad(i=1,2)$,

Where $E$ represents the material elastic modulus, $d_{i}$ and $m_{i}$ represents the maximum displacement and structural mass, $i$ represents the different bionic model.

The stiffness efficiency of four bionic models is calculated by Eq. (7) and normalized to $\varepsilon$ by Eq. 8, the results are shown in Table 3. When $\varepsilon=1$, the stiffness efficiency of the bionic model is the highest.

$\varepsilon=\varepsilon_{i} / \varepsilon_{\max }$,

It can be seen from Table 3 that all the four bionic prototypes have good pressure-resistance performance, and the cross-section of wheat-stalk has good bending-resistance and torsion-resistance. When the crosssection of skeleton structure bears bending moment and torsion load, wheat-stalk is preferred as biological prototype. From the perspective of natural environment, the wheat-stalk not only bears the weight of wheat ear, but also bear the lateral wind force.

Table 3. The stiffness efficiency ratio under different load type

\begin{tabular}{ccccc}
\hline & Bamboo & wheat-stalk & Juncus & $\begin{array}{c}\text { Brazilian } \\
\text { Horsetail }\end{array}$ \\
\hline Pressure-resistance & 1 & 1 & 1 & 1 \\
Bending-resistance & 0.95 & 1 & 0.96 & 0.97 \\
Torsion-resistance & 0.94 & 1 & 0.87 & 0.85 \\
\hline
\end{tabular}




\section{Bionic design and discussion}

According to Section 3.3, the coupling load of bending moment and pressure is the load-bearing type for the new control arm. So, the cross-section with good bending-resistance and torsion-resistance should be selected as the biological prototype. Among the several bionic prototypes provided in this paper, the wheatstalk has good mechanical performance and matches with the skeleton of control arm. So, a wheat-stalk-based bionic model is design as shown in Figure 16 . The weight is reduced to $0.68 \mathrm{~kg}$, which is $32.7 \%$ lighter than the original model.

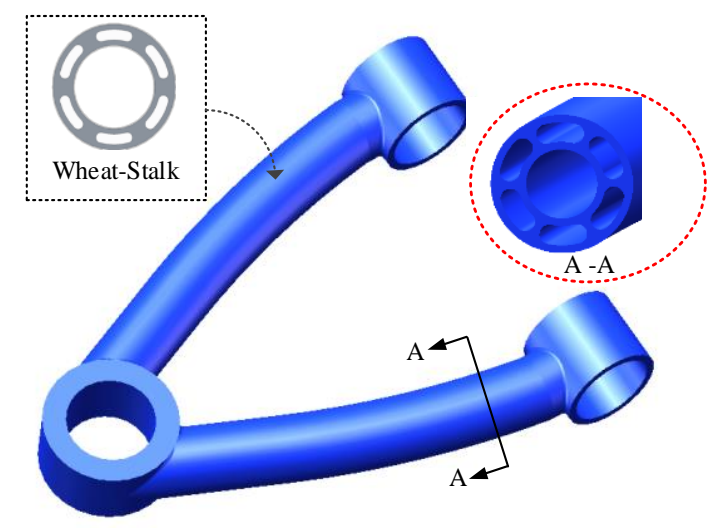

Figure 16. The bionic model of control arm

To verify the mechanical performance of the bionic model of the control arm., the FEA is firstly conducted to check the structural stiffness and strength of the original model (Figure 2), improved model (Figure 11) as well as bionic model (Figure 16). Applying the same load and boundary conditions to the three models, and the Finite Element Models are established with same element size and type. The FEA results are shown in Table 4.

Table 4. The FEA results of three models

\begin{tabular}{|c|c|c|}
\hline & Displacement distribution & Stress distribution \\
\hline Original model & 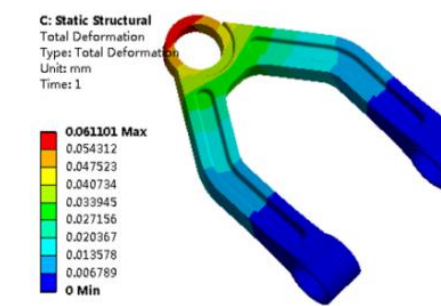 & 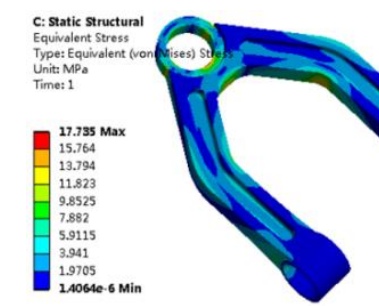 \\
\hline $\begin{array}{c}\text { Improved } \\
\text { model }\end{array}$ & 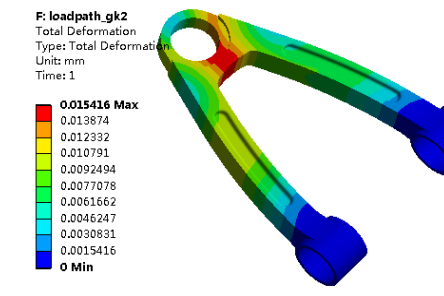 & 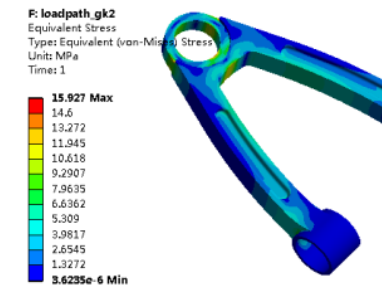 \\
\hline Bionic model & 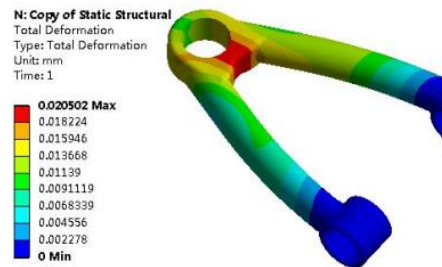 & 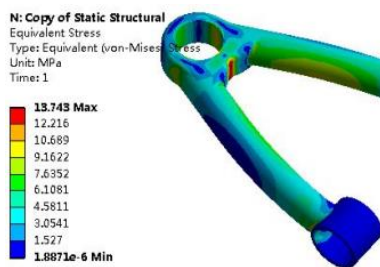 \\
\hline
\end{tabular}

The maximum displacement and stress of three models are compared in Table 5. The maximum displacement 
of the bionic model is $0.021 \mathrm{~mm}$, which reduces $0.04 \mathrm{~mm}$ compared with the original model. The maximum stress is $13.74 \mathrm{MPa}$, which is far less than the material yield strength (220 MPa), and the stress distribution is more uniform than the original model.

Table 5. Mechanical properties comparison of the control arm

\begin{tabular}{cccc}
\hline & mass $(\mathrm{kg})$ & Max displacement(mm) & Max stress (MPa) \\
\hline Original model & 1.01 & 0.061 & 17.74 \\
Improved model & $0.92(-8.9 \%)$ & $0.015(-75.4 \%)$ & $15.93(-10.2 \%)$ \\
Bionic model & $0.68(-32.7 \%)$ & $0.021(-65.6 \%)$ & $13.74(-22.5 \%)$ \\
\hline
\end{tabular}

From the above analysis, it can be concluded that the model modification by load path can improve the structural load-transfer performance. The application of cross-section of wheat-stalk further realize the structural lightweight. The stiffness and strength of the control arm are improved by $65.6 \%$ and $22.5 \%$ respectively, and its weight is reduced by $32.7 \%$. The results show that the proposed method can provide better topological structure for the skeleton structures.

\section{Conclusions}

In this study, a new design method of control arm combining load path and biological characteristics is proposed. There are three steps including: load path analysis and improvement, construct biological crosssection, and bionic design. The load path is a effective tool to visualize the load-transferred law in structure and give structural improvement suggestions, also can make clear and modify the structural load-bearing types. Four kinds of plants including Bamboo, Wheat-stalk, Juncus, Brazilian Horsetail are selected as biological prototypes of control arm. The load-bearing type is analyzed by Finite element analysis and the cross-section of wheat-stalk has good bending-resistance and torsion-resistance, which is selected as the cross-section of control arm. The bionic model is designed by applying the cross-section of wheat-stalk to the improved control arm. The results show that the stiffness and strength of the control arm are improved by $65.6 \%$ and $22.5 \%$ respectively, and its weight is reduced by $32.7 \%$, which show that the proposed method can provide better topological structure for the skeleton structures.

Acknowledgement: This research is supported by Scientific and Technological Innovation Programs of Higher Education Institutions in Shanxi (2021L291), Science and Technology Project of Hebei Education Department (ZD2020156), and Doctoral Research Startup Fund Project of Taiyuan University of Science and Technology (20212040).

\section{References}

Cai, K. \& Shi, J. (2010) A bionic approach for topology optimization for tension-only or compression-only design, Journal of Bionic Engineering. 7(4), 397-404. https://doi.org/10.1016/S1672-6529(10)60272-7

Cheng, L., Bai, J.X. \& To, A.C. (2019) Functionally graded lattice structure topology optimization for the design of additive manufactured components with stress constraints, Computer Methods in Applied Mechanics and Engineering, 344, 334-359. https://doi.org/10.1016/j.cma.2018.10.010

Dong, Y.J. \& Zhu, G.W. (2016) Mechanical analysis and bionic structure design of astronautic payloads based on natural honeycomb, Journal of Astronautics. 37, 262-267. http://www.yhxb.org.cn/EN/Y2016/V37 /I3/262

Kakoty, N.M. \& Hazarika, S.M. (2013) A biomimetic similarity index for prosthetic hands, In Proceedings of the IEEE Symposium on Computational Intelligence in Rehabilitation and Assistive Technologies, Singapore, 32-39. https://doi.org/10.1109/CIRAT.2013.6613820

Heo, S.J., Kang, D.O., Lee, J.H., Kim, I.H. \& Darwish, S.M.H. (2013). Shape optimization of lower control arm considering multi-disciplinary constraint condition by using progress meta-model method. International Journal of Automotive Technology, 14(3), 499-505. https://doi.org/10.1007/s12239-013-0054-7

Pejhan, K., Kuznetcov, A., Wang, Q.G., Wu, C.Q., \& Telichev, Igor. (2017). Design assessment of a multiple passenger vehicle component using load transfer index $\left(\mathrm{U}^{*}\right)$ method. International journal of mechanics \& materials in design. 14(2), 1-17. https://doi.org/10.1007/s10999-017-9372-7 
Suzuki, T., Fukushige, S. \& Tsunori, M. (2020) Load path visualization and fiber trajectory optimization for additive manufacturing of composites, Additive Manufacturing. 2020, 31:100942. https://doi.org/10.1016/j. addma.2019.100942

Takahashi, K. (1986) Relative rigidity of structures and Saint Venant's principle. Transactions of the Japan Society of Mechanical Engineers Series A. 52, 2615-21. https://doi.org/10.1299/kikaia.52.2615

Takahashi, K., Omiya, M., Iso, T., Zaiki, Y., Sakurai, T., Maki, T., Urushiyama, Y. \& Naito, T. (2013). Load transfer ustar $\left(\mathrm{U}^{*}\right)$ calculation in structures under dynamic loading. Transactions of the Japan Society of Mechanical Engineers, 79(807), 1657-1668. https://doi.org/10.1299/kikaia.79.1657

Viqaruddina, M. \& Ramana R.D. (2017). Structural optimization of control arm for weight reduction and improved performance. Materials today: proceedings, 4(8), 9230-9236. https://doi.org/10.1016/j.matpr. 2017.07.282

Wang, C.Y., Li, Y., Zhao, W.Z., Zou, S.C., Zhou, G. \& Wang, Y.L. (2018) Structure design and multi-objective optimization of a novel crash box based on biomimetic structure, International Journal of Mechanical Sciences. 138, 489-501. https://doi.org/10.1016/j.ijmecsci.2018.01.032

Yoo, S.H., Doh, J. \& Lim, J. (2017). Topologically optimized shape of CFRP front lower control ARM. International Journal of Automotive Technology, 18(4), 625-630. https://doi.org/10.1007/s12239-017-0062-0

Wang, Q.G., Pejhan, K., Telichev, I., Wu, C.Q. (2018) Demonstration of the effectiveness of U*-based design criteria on vehicle structural design. Proceedings of the Institution of Mechanical Engineers Part D Journal of Automobile Engineering, 232(8), 995-1002. https://doi.org/10.1177/0954407017724635

Waldron, D. \& Harwood J. (2010) A study of the relationship between bending rigidity and the ease of decortication of flax straw. Journal of Natural Fibers, 7(1), 42-60. https://doi.org/10.1080/1544047090 3579226

Wang, Z.H., Wu, N., Wang, Q.G., Li, Y.X., Yang, Q.W. \& Wu, F.H. (2020) Novel bionic design method for skeleton structures based on load path analysis. Applied sciences. 8251(10), 1-17. https://doi.org/10.3390/app 10228251

Wang, Z.H., Wang Q.G., Wu, N., Guo, BS. \& Wu F.H. (2021) Structural improvement of vehicle component based on the load path and load distribution analysis. International Journal of Automotive Technology. 22, 787 798. https://doi.org/10.1007/s12239-021-0072-9

Zhang, T.C., Wang, A.L., Wang, Q.S. \& Guan, F.R. (2019) Bending characteristics analysis and lightweight design of a bionic beam inspired by bamboo structures, Thin-Walled Structures. 142, 476-498. https://doi.org/ 10.1016/j.tws.2019.04.043

Zhang, Z.F., Chen, R., Xu Z.M., He, Y.S. \& Li, W. (2017) Research on multi-objective topology optimization of vehicle suspension control arm. Journal of mechanical engineering, 53(04), 114-121. (in chinese) https:// doi.org/ 10.3901/JME.2017.04.114

Zhao, L., Ma, J., Chen, W., \& Guo, H. (2011) Lightweight design and verification of gantry machining center crossbeam based on structural bionics, Journal of Bionic Engineering. 8, 201-206. https://doi.org/10.1016/ S1672-6529 (11)60021-8 